\title{
Real time RT-PCR assays for detection and genotyping of West Nile Virus lineages circulating in Africa.
}

Gamou Fall (gamou.fall@pasteur.sn), Martin Faye ${ }^{1}$ (martin.faye@pasteur.sn), Manfred Weidmann² (m.w.weidmann@stir.ac.uk), Marco Kaiser ${ }^{3}$

(Kaiser@genexpress.de), Anne Dupressoir ${ }^{1}$ (dupresso@igr.fr), El Hadj Ndiaye ${ }^{4}$ (elndiaye@pasteur.sn), Yamar Ba ${ }^{4}$ (ba@pasteur.sn), Mawlouth Diallo ${ }^{4}$ (diallo@pasteur.sn), Ousmane Faye ${ }^{1}$ (ofaye@pasteur.sn), Amadou Alpha Sall ${ }^{1}$ (asall@pasteur.sn)

1. Unité des arbovirus et virus de fièvres hémorragiques, Institut Pasteur de Dakar, BP220 Senegal

2. Institute of Aquaculture, University of Stirling, Stirling FK9 4LA, UK

3. GenExpress GmbH, Berlin, Germany

4. Unité d'Entomologie médicale, Institut Pasteur de Dakar, BP220 Senegal

Publisher policy allows this work to be made available in this repository. Published in Vector-Borne and Zoonotic Diseases, December 2016, 16(12): 781-789 by Mary Ann Liebert, Inc. The original publication is available at: https://doi.org/10.1089/vbz.2016.1967

Keywords: West Nile virus, African lineages, Real time RT-PCR, genotyping 


\section{Introduction}

West Nile virus (WNV) is a mosquito-borne virus belonging to the Japanese encephalitis complex of the Flaviviridae family including Usutu virus (USUV), Japanese encephalitis virus (JEV), Saint Louis encephalitis virus (SLEV) and Murray Valley Fever virus (MVFV) (Gubler et al., 2007). WNV was first isolated and identified in the West Nile region of Uganda in 1937 from a febrile female adult patient (Smithburn et al., 1940). WNV is transmitted by the bite of infected mosquitoes, particularly Culex spp mosquitoes. Birds are considered as a main reservoir host and migratory birds and can play an important role in long distance viral spread (Hubalek and Halouzka, 1999; Hayes et al., 2005, Ergunay et al., 2015). West Nile fever outbreaks concern essentially humans, and horses as dead-end hosts (Hubalek and Halouzka, 1999; Hayes et al., 2005; Kulasekera et al., 2001; Murray et al., 2010). Clinical symptoms range from asymptomatic or mild influenza-like illness to severe neurological disease, which can be characterized by acute flaccid paralysis, encephalitis and meningoencephalitis (Hayes et al., 2005; De Filette et al., 2012). Since the mid-1990s, WNV outbreaks have emerged in Europe and America and WNV is now recognized as one of the most widespread flaviviruses worldwide (Vilibic-Cavlek et al., 2014; Hernández-Triana et al., 2014; DeGroote et al., 2014; Cox et al., 2015) and not restricted to tropical regions.

WNV exhibits a great genetic diversity with at least eight different lineages circulating in the world, without known specific biological properties (Mackensie \& Williams, 2009; Fall et al., 2014). Among them, 4 (i.e. 1, 2, Koutango and a putative new lineage) are present in Africa, particularly in Senegal. Lineage 1 is the only one found worldwide, and associated with all major human outbreaks (Murray et al., 2010; Hernández-Triana et al., 2014; Anukumar et al., 2014). 
Lineage 2 was exclusively present in Africa but since 2004, this lineage has been reported circulating in Europe, with severe cases in humans and birds (Hernández-Triana et al., 2014; Bakonyi et al., 2006). Viruses with high and low neuro-invasiveness phenotype exist in both lineages 1 and 2. Koutango and the putative new lineage are local African lineages, never isolated in humans, birds or horses (Fall et al., 2014; http://www.pasteur.fr/recherche/banques/CRORA/). Recently, the putative new lineage was experimentally shown to be transmitted by Culex neavei, which suggests a possible transmission particularly to birds, and horses (Fall et al., 2014). The Koutango lineage was reported more neurovirulent in mice than WNV NY99, a known virulent lineage 1 strain (Prow et al., 2014). These findings provided new insights into transmission and pathogenesis of these African local WNV lineages.

The report of lineage 2 in Europe, which was a local African lineage, suggests ongoing exchanges between Africa and Europe, and migratory birds that overwintered in Africa may have introduced lineage 2 into Europe (Bakonyi et al., 2006; Hernández-Triana et al., 2014). A similar migration event has been also observed for Usutu virus (Nikolay et al., 2013).

Interestingly, Senegal is an important stopover point for bird migration between Africa and Europe in the area of the ornithological park Djoudj on the Senegal river. All these observations suggest the potential of the African local lineages (Koutango and the putative new lineage) to spread beyond their expected geographical areas into Europe.

These different WNV lineages are not readily differentiated by serology; therefore, rapid molecular methods are required for detection and genotyping of WNV in suspect cases and potential vectors. This may have important applications in surveillance and epidemiology of African WNV lineages in Africa but also for surveillance of their emergence in Europe and other 
continents. Molecular tools are available for WNV detection (Linke et al., 2007; Zaayman et al., 2009; Faggioni et al., 2014) but the genetic diversity of all African WNV variants has never been taken into account. Here we developed rapid molecular tools for a consensus detection of all WNV lineages (WNV consensus assay) and genotyping of the different WNV lineages reported in Africa (lineage-specific assays).

\section{Materials and methods}

\section{Virus}

Fifteen WNV and 7 other flavivirus isolates were used in this study and are described in table 1. The virus stocks were prepared by inoculating Aedes pseudoscutellaris (AP61) continuous cell lines for 4 days and Immuno-Fluorescence Assay (IFA) as previously described (Digoutte et al., 1992) was performed to assess the cell infection with WNV. Virus stocks were titrated as

previously described, using PS cells (Porcine stable kidney cell line, American Type Culture Collection, Manassas, USA) (De Madrid AT and Porterfield JS, 1969). The cell culture media were used as viral stocks for RNA extraction and RT-PCR.

\section{Primers and probes design for WNV consensus assay}

In order to develop a LNA (Locked Nucleic Acid)-probe based real time RT-PCR, target regions described previously, envelope gene (E-gene) (Lanciotti et al.,1999) and 3'UTR (Clavero et al., 2006) were considered. The Envelope gene target was dismissed as it had been chosen for detection of American WNV type 1 sequences only. Using 78 sequences available from Genbank at the time of design, an amplicon was designed for the 3'UTR target region. Because of too high divergence the sequences "Rabensburg" (AY765264) and "LEIV-Krnd88-190" (AY277251) were not included in the alignments. These primers were synthesized (TIB Mol-Biol, Berlin, 
Germany) and tested to identify a pair, which would not amplify any of the other Flaviviruses tested (table 2).

\section{Primers and probes design for WNV lineage-specific assays}

In order to develop lineages specific-real time RT-PCR assays, the E-gene sequences of strains belonging to each lineages $(1,2$, Koutango and the putative new lineage) were considered. The different primers and probes designed (table 2) were synthesised (TIB Mol-Biol, Berlin, Germany) and tested.

\section{RNA standard}

For the consensus assay, a 1047 nucleotide fragment of the 3'UTR region was amplified using WNVUTR UP 5'-TGCTTCTGTACTTCCACAGAAGAG-3 and WNVUTR DP 5'AGATCCTGTGTTCTCGCACC-3. For the lineage-specific assays, 727bp fragments of the Egene were amplified with the primers WNV E-F (5'-CAACTGCCTAGGAATGAGYAACAG3') and WNV E-R (5'-GGCATGAGGTTCTTCAAACTC CA-3'). The obtained PCR products were ligated into pCRII (3'UTR) and pCR2.1 (E-gene) (Life Technologies, GmbH, Darmstadt) and used for in vitro transcription of an RNA standard with T7 RNA-polymerase (Roche, Mannheim, Germany) as described previously (Nikolay et al., 2014).

\section{Determination of specificity}

The specificity of the different assays was determined by testing $10 \mathrm{WNV}$ strains belonging to the 4 lineages circulating in Africa (i.e 3 lineage-1, 4 lineage-2, 2 Koutango, and the unique strain of the putative new lineage) in comparison to other flaviviruses isolates of Dengue virus, Usutu virus, Yellow fever virus, Zika virus and Bagaza. Prior to WNV RT-PCR assays, all these isolates were tested using Pan-Flavi assay (Patel et al., 2013) and existing specific assays for 
DENV (Wagner et al., 2004), YFV (Weidmann et al., 2010), and Zika (Faye et al., 2013) as described previously for amplification control.

\section{Determination of sensitivity}

Ten-fold dilutions of each in vitro RNA standard with known initial copy number were quantified in triplicate using the different assays. Regression curves were obtained representing the RNA copy number/reaction vs the threshold cycle value $(\mathrm{Ct})$. The lowest RNA copy number with RTPCR detection was considered as the analytical detection limit.

The sensitivity of viral RNA detection was also evaluated by serial 10 -fold dilutions of viral stocks with known titer of each WNV type in L-15 medium (Gibco BRL, GrandIsland, NY, USA) and in human serum (Sigma Aldrich, Saint-Louis, MO, USA). To verify for the number of genome copies per plaques forming units (pfu) all supernatants were quantified using the respective WNV real time RT-PCR assays. The WNV reference strain Eg101 was used for lineage 1-specific and consensus RT-PCR assays. The WNV reference strains B956, ArD96655, and ArD94343 were used respectively for lineage 2, Koutango, and new lineage-specific assays. The assays were performed in triplicate. For the 10 -fold dilutions, regression curves were obtained representing the pfu/reaction vs the threshold cycle value $(\mathrm{Ct})$. The lowest titer with RTPCR detection was considered as the analytical detection limit.

\section{Processing of field samples and experimentally infected mosquitoes}

Field mosquito pools and rodents, and experimentally infected mosquitoes (Fall et al., 2014) were used to test the performance of the assays. Experimentally infected mosquitoes were homogenized as previously described (Fall et al., 2014) and mosquito pools collected in the field were homogenized in $3 \mathrm{ml}$ of L-15 supplemented with $20 \%$ of foetal bovine serum FBS. Rodent 
tissues were homogenized in 3 to $4 \mathrm{ml}$ of L-15 containing $5 \%$ FBS. Viral RNA was extracted from the homogenates and analyzed by real time RT-PCR assays (see below).

\section{RNA extraction and RT-PCR}

RNA was extracted from $100 \mu \mathrm{l}$ of each viral stock, mosquito or rodent homogenates and 10-fold dilution of WNV lineage stocks in L-15 medium or human serum using the QiaAmp Viral RNA extraction kit (Qiagen, Heiden, Germany) according to the manufacturer's instructions.

The real-time RT-PCR assay was performed using an ABI 7500 cycler (Applied Biosystems, Foster City, US) and the Quantitect Probe RT-PCR kit (Qiagen, Heiden, Germany). Reactions were performed in $25 \mu \mathrm{l}$ reaction volume containing $1 \mu \mathrm{l}$ diluted RNA, $0.5 \mu \mathrm{M}$ foward and reverse primer, $0.2 \mu \mathrm{M}$ of probe, $12.5 \mu \mathrm{l}$ of $2 \times$ QuantiTect Probe RT-PCR Master Mix, and 0.25 $\mu 1$ of Quantitect RT Mix. The RT-PCR conditions were as follows: $15 \min 50^{\circ} \mathrm{C}, 10 \mathrm{~min} 95^{\circ} \mathrm{C}$ and 40 cycles of $15 \mathrm{~s} 95^{\circ} \mathrm{C}$ and $1 \mathrm{~min} 60^{\circ} \mathrm{C}$.

\section{Determination of intra, inter-assay reproducibility and efficiency}

Samples were extracted and amplified 10 times in the same run to evaluate intra-assay variability and in 10 different runs to evaluate inter-assay variability. The amplification efficiency of the primers was calculated from the slope of the standard regression lines $\left(E=10^{1 / \text { slope }}-1\right)$.

\section{Results}

\section{Analytical specificity and analytical sensitivity}

RNAs from the $10 \mathrm{WNV}$ and other flaviviruses isolates were tested using Pan-Flavi, WNV consensus, and WNV lineage-specific real time RT-PCR assays developed herein. The Pan-Flavi assay detected all the isolates tested. The WNV consensus assay detected all the $10 \mathrm{WNV}$ strains but initially also detected RNAs from Usutu virus strains (data not shown). Using the ARMS (amplification refractory mutation system) principle (Newton et al., 1989; Weidmann et al., 
2003) the reverse primer was adapted to an insertion in the USUV sequences matching the $2^{\text {nd }}$ position of a newly designed WNV reverse primer (figure 1). The new WNV consensus assay (table 2) now did not show any more cross detection of RNAs from Usutu virus strains.

To boost differentiation and therefore specificity of the individual WNV lineage-specific assays, primers were also designed using the ARMS principle with specific matches at the 2-3 position of the 3 '-end of the primers mismatching to as many of the other strains as possible (figure 2). These lineage-specific assays detected and correctly genotyped the respective WNV strains tested in each lineage and did not detect other flaviviruses tested nor cross-react within other WNV lineages tested (table 3).

The performance of the assays was tested by using 10 -fold dilutions of in vitro RNA standards. Five independent runs were done using the RNA standards of the consensus and each of the lineage-specific assays (figure 3). The analytical detection limit was 10 copies/reaction for all the RT-PCR assays (figure 3). The linear regression showed that the different curves were linear with correlation coefficients $\geq 0.998$ in all cases. Efficiencies ranged from $99 \%$ to $141 \%$ with lower efficiencies for Koutango (103\%) and putative new lineage (99\%) assays, and higher efficiency for the consensus assay (141\%). The lineages 1 and 2 assays were intermediate with $129 \%$ and $121 \%$ respectively (table 4 ).

\section{Viral RNA quantification}

The determined titers of Eg101, B956, ArD96655 and ArD94343 (corresponding respectively to lineages 1, 2, Koutango and new lineage viral stocks) are presented table 4. The RT-PCR analyses on these viral stocks were done using lineage-specific assays and the copy number and ratio copy number/pfu are presented table 4 . 
Ten-fold dilutions of Eg101, B956, ArD96655, and ArD94343 prepared in L-15 medium and human serum were analyzed by RT-PCR with the lineage-specific assays. The consensus assay was tested only on strain Eg101 (lineage 1) and yielded a detection limit of $0.13 \mathrm{pfu} / \mathrm{reaction}$ (Figure 4A). The lineage specific reactions yielded a detection limit of $1.3 \mathrm{pfu} /$ reaction, 5 pfu/reaction, $4.49 \mathrm{pfu} /$ reaction and $9.16 \mathrm{pfu} /$ reaction respectively for the detection of lineage 1 , lineage 2, Koutango and the putative new lineage (figures 4B-E).

The curves obtained were comparable between L-15 and human serum and linear with correlation coefficients $\geq 0.998$. Efficiencies ranged from $74 \%$ to $158 \%$ for dilutions in L15, and $82 \%$ to $138 \%$ for dilutions in human serum, with higher efficiencies for the consensus assay, and lower efficiencies for lineage 2-specific assay in both L15 and human serum.

\section{Processing of field samples and experimentally infected mosquitoes}

To assess the performance of our assays, a panel of samples from experimentally infected mosquitoes, wild caught mosquito pools, and rodent tissues were used. For each experimental sample, the corresponding virus used to infect the mosquitoes was correctly detected and genotyped (table 5). The field samples naturally infected with WNV lineage 1 or Koutango, as

previously assessed by classical RT-PCR and sequencing (data not shown), were correctly detected and genotyped with the lineage-specific assays in the mosquito pools or rodent tissues including brain homogenates (table 5).

\section{Variability intra and inter-assay}

The intra-assay coefficients variation $(\mathrm{CV})$ ranged from 1.31 to $1.75 \%$ and the inter-assay $\mathrm{CV}$ ranged from 2.66 to $4.62 \%$ for all assays. 


\section{Discussion}

Molecular techniques are faster, more accurate and sensitive for virus detection compared to culture methods. Here, we established molecular tools for a consensus detection of all WNV lineages and genotyping of the lineages reported in Africa. The good sensitivity of the assays allows the detection and genotyping of all WNV isolates in culture medium, human serum, as well as in field and experimental samples. After the development of the lineage specific assays new WNV lineage 2 sequences described in Europe became available (Barzon et al., 2013). Since they introduce a mismatch at position 3 from the 3 'end of the lineage 2 specific forward primer, we suggest to use the lineage 1-specific upstream primer which is also used for Koutango for highly sensitive detection of these strains (Figure 2). However, we were not able to test these strains in the frame of this study.

Our assays allowed the quantification of WNV RNA by using quantitative RNA standards, yielding viral loads in mosquito and vertebrate samples.

Using the RNA standard, the different assays were very sensitive and able to detect 10 RNA molecules. Our RT-PCR assays exhibit therefore analytical sensitivity similar to others using WNV lineages 1 and 2 (Clavero et al., 2006; Barros et al., 2013; Del Amo et al., 2013).

Using viral RNA, the consensus and lineage 1- specific assay were more sensitive and were able to detect less than 2 pfu of virus. Using the same strain for the consensus and lineage 1-specific assays showed that the consensus assay is more sensitive for detection of low viral loads. This is reflected by the different ratios copy number/pfu obtained for Eg101 (56.08 with the consensus assay and 34.34 with the lineage 1-specific assay) and can be correlated with the higher efficiencies of the consensus RT-PCR assay. 
Our results showed also that the different WNV lineages can be detected in human serum with slightly higher efficiencies compared to culture medium, and in vertebrate tissues, enabling the early and efficient diagnosis of these different WNV lineages from clinical samples. Finally our study revealed finally new insight on the replication of these particular WNV strains in AP61 mosquito cells. The ratios genome copy number / infectious virion (pfu) showed that new lineage strain and Koutango replication were very efficient with few genomes per pfu indicating apparently a high rate of infectious particles produced, while lineage 2 appeared to overproduce genomes indicating less efficient packaging and possibly more defective particles during its replication (Weidmann et al., 2011).

\section{Conclusions}

To our knowledge, the RT-PCR assays described in this paper are the first ones that allow the detection and genotyping of all reported African WNV variants.

Surveillance programs now can target all WNV variants, and our tools can be used to monitor the prevalence of WNV virus lineages in mosquito vectors and vertebrates and may be applied in diagnosis as well as in epidemiology and surveillance programs. This is particularly relevant for the surveillance of WNV lineages emerging in geographic regions where they have not previously been identified. The consensus assay is the most sensitive in all tested materials and does not cross detect any of the tested Flaviviruses and therefore is an ideal diagnostic assay covering the breadth of WNV strains worldwide.

Therefore, our tools are efficient for rapid detection of African WNV lineages, however they cannot replace conventional techniques (serology, virus isolation or molecular sequencing), which are still needed for in-depth characterization of the virus. 


\section{Acknowledgements}

\section{Technical support}

The authors thank Moussa Dia, Mireille Mondo, Arame Ba and Khardiata Mbaye for their excellent technical assistance.

\section{Financial support}

This work has been financially supported by Institut Pasteur de Dakar, and funded by EU grant HEALTH.2010.2.3.3-3-261391 EuroWestNile.

\section{Author Disclosure Statement}

No competing financial interests exist.

\section{References}

1) Anukumar B, Sapkal GN, Tandale BV, Balasubramanian R, et al. West Nile encephalitis outbreak in Kerala, India, 2011. J Clin Virol 2014; 61(1):152-155. 
2) Bakonyi T, Ivanics E, Erdélyi K, Ursu K, et al. Lineage 1 and 2 strains of encephalitic West Nile virus, central Europe. Emerg Infect Dis 2006; 12(4):618-623.

3) Barros SC, Ramos F, Zé-Zé L, Alves MJ, et al. Simultaneous detection of West Nile and Japanese encephalitis virus RNA by duplex TaqMan RT-PCR. J Virol Methods 2013; 193(2):554-557.

4) Barzon L, Pacenti M, Franchin E, Lavezzo E, Masi G, Squarzon L, Pagni S, Toppo S, Russo F, Cattai M, Cusinato R, Palu G. Whole genome sequencing and phylogenetic analysis of West Nile virus lineage 1 and lineage 2 from human cases of infection, Italy, August 2013. Euro Surveill 2013 Sep 19;18(38).

5) Cox SL, Campbell GD, Nemeth NM. Outbreaks of West Nile virus in captive waterfowl in Ontario, Canada. Avian. Pathol 2015; 30:1-27.

6) De Filette M, Ulbert S, Diamond M, Sanders NN. Recent progress in West Nile virus diagnosis and vaccination. Vet Res 2012; 43:16.

7) DeGroote JP, Sugumaran R, Ecker M. Landscape, demographic and climatic associations with human West Nile virus occurrence regionally in 2012 in the United States of America. Geospat Health 2014; 9(1):153-168.

8) Del Amo J, Sotelo E, Fernández-Pinero J, Gallardo C, et al. A novel quantitative multiplex real-time RT-PCR for the simultaneous detection and differentiation of West Nile virus lineages 1 and 2, and of Usutu virus. J Virol Methods 2013; 189(2):321-327.

9) De Madrid AT, Porterfield JS. A simple micro-culture method for the study of group B arboviruses. Bull World Health Organ 1969; 40:113-121. 
10)Digoutte JP, Calvo-Wilson MA, Mondo M, Traore-Lamizana M, et al. Continuous cell lines and immune ascitic fluid pools in arbovirus detection. Res Virol 1992; 143(6):417422.

11)Ergunay K, Bakonyi T, Nowotny N, and Ozkul A. Close Relationship between West Nile Virus from Turkey and Lineage 1 Strain from Central African Republic. Emerg Infect Dis $2015 ; 21(2): 352-355$.

12)Faggioni G, De Santis R, Pomponi A, Fantini M, et al. Rapid molecular detection and genotyping of West Nile Virus lineages 1 and 2 by real time PCR and melting curve analysis. J Virol Methods 2014; 207:54-59.

13) Fall G, Diallo M, Loucoubar C, Faye O, et al. Vector Competence of Culex neavei and Culex quinquefasciatus (Diptera: Culicidae) for lineages 1, 2, Koutango and a putative new lineage of West Nile Virus from Africa. Am J Trop Med 2014; 90(4):747-754.

14) Faye O, Faye O, Diallo D, Diallo M, et al. Quantitative real-time PCR detection of Zika virus and evaluation with field-caught mosquitoes. Virol J 2013; 10:311.

15) Gubler DJ. The continuing spread of West Nile virus in the western hemisphere. Clin Infect Dis 2007; 45(8):1039-1046.

16) Hayes EB, Sejvar JJ, Zaki SR, Lanciotti RS, Bode AV, Campbell GL. Virology, pathology, and clinical manifestations of West Nile virus disease. Emerg Infect Dis 2005; 11(8):1174-1179.

17)Hernández-Triana LM, Jeffries CL, Mansfield KL, Carnell G, et al. Emergence of west nile virus lineage 2 in Europe: a review on the introduction and spread of a mosquitoborne disease. Front Public Health 2014; 2:271. 
18) Hubalek $Z$ and Halouzka J. West Nile fever a reemerging mosquito-borne viral disease in Europe. Emerg. Infect. Dis 1999; 5:643-650.

19) Jiménez-Clavero MA, Agüero M, Rojo G, Gómez-Tejedor C. A new fluorogenic realtime RT-PCR assay for detection of lineage 1 and lineage 2 West Nile viruses. J Vet Diagn Invest 2006; 18(5):459-462.

20) Kulasekera VL, Kramer L, Nasci RS, Mostashari F, Cherry B, Trock SC, et al. West Nile virus infection in mosquitoes, birds, horses, and humans, Staten Island, New York, 2000. Emerg Infect Dis 2001; 7(4):722-725.

21)Lanciotti RS, Kerst AJ, Nasci RS, Godsey MS, et al. Rapid detection of west nile virus from human clinical specimens, field-collected mosquitoes, and avian samples by a TaqMan reverse transcriptase-PCR assay. J Clin Microbiol 2000; 38(11):4066-4071.

22)Linke S, Ellerbrok H, Niedrig M, Nitsche A, et al. Detection of West Nile virus lineages 1 and 2 by real-time PCR. J Virol Methods 2007; 146(1-2):355-358.

23) Mackenzie JS, Williams DT. The zoonotic flaviviruses of southern, south-eastern and eastern Asia, and Australasia: the potential for emergent viruses. Zoonoses Public Health $2009 ; 56(6-7): 338-356$.

24)Murray KO, Mertens E, Despres P. West Nile virus and its emergence in the United States of America. Vet Res 2010; 41(6): 67.

25)Newton CR, Graham A, Heptinstall LE, Powell SJ, et al. Analysis of any point mutation in DNA. The amplification refractory mutation system (ARMS). Nucleic Acids Res 1989; $17: 2503-2516$

26) Nikolay B, Dupressoir A, Firth C, Faye O, et al. Comparative full length genome sequence analysis of Usutu virus isolates from Africa. Virol J 2013; 10:217. 
27) Nikolay B, Weidmann M, Dupressoir A, Faye O, et al. Development of a Usutu virus specific real-time reverse transcription PCR assay based on sequenced strains from Africa and Europe. J Virol Methods 2014; 197:51-54.

28) Patel P, Landt O, Kaiser M, Faye O, et al. Development of one-step quantitative reverse transcription PCR for the rapid detection of flaviviruses. Virol J 2013; 10:58.

29) Prow NA, Setoh YX, Biron RM, Sester DP, et al. The West Nile virus-like flavivirus Koutango is highly virulent in mice due to delayed viral clearance and the induction of a poor neutralizing antibody response. J Virol 2014; 88(17):9947-9962.

30) Smithburn KC, Hughs TP, Burke AW, Paul JH. A neurotropic virus isolated from the blood of a native of Uganda. Am J Trop Med 1940; 20:471-492.

31)Vilibic-Cavlek T, Kaic B, Barbic L, Pem-Novosel I, Slavic-Vrzic V, Lesnikar V, et al. First evidence of simultaneous occurrence of West Nile virus and Usutu virus neuroinvasive disease in humans in Croatia during the 2013 outbreak. Infection 2014; 42(4):689-695.

32) Wagner D, de With K, Huzly D, Hufert F, et al. Nosocomial acquisition of dengue. Emerg Infect Dis 2004; 10(10):1872-1873.

33) Weidmann M, Faye O, Faye O, Kranaster R, et al. Improved LNA probe-based assay for the detection of African and South American yellow fever virus strains. J Clin Virol 2010; 48(3):187-192.

34) Weidmann M, Rudaz V, Nunes MR, Vasconcelos PF, et al. Rapid detection of human pathogenic orthobunyaviruses. J Clin Microbiol 2003; 41(7):3299-3305. 
35) Weidmann M, Sall AA, Manuguerra JC, Koivogui L, et al. Quantitative analysis of particles, genomes and infectious particles in supernatants of haemorrhagic fever virus cell cultures. Virol J 2011; 8:81.

36)Zaayman D, Human S, Venter M. A highly sensitive method for the detection and genotyping of West Nile virus by real-time PCR. J Virol Methods 2009; 157(2):155-160.

\section{Correspondence address}

\section{Dr Amadou Alpha Sall}

Unité des Arbovirus et virus de fièvres hémorragiques,

Institut Pasteur de Dakar, BP 220 Dakar, SENEGAL

Phone: +221.338399223

Fax: +221.338399210

Email: asall@pasteur.sn 


\section{Figure legends}

Figure 1: Sequence alignment of 3'UTR sequences of WNV and USUV as indicated by accession numbers with WNV consensus assay primers and LNA-probe. Nucleotide positions refer to the sequence of reference strain Eg101 from Egypt (AF260968). AF196835 represents sequences from the USA. AY277252 represents sequences from Russia.

Figure 2: Sequence alignment of WNV E gene sequences from Africa, Senegal, Egypt, Uganda, Europe and America as indicated by strain designations with WNV lineage specific primers and probes. The forward primer WNV FP is common to lineages 1, Koutango and the European WNV2 isolates. Nucleotide positions refer to the sequence of reference strain Eg101 from Egypt (AF260968). Probe mismatches are highlighted in bold in reference to probe WNV1 P for the detection of WNV lineage 1. Mismatches introduced to the 3' ends of the primers according to the ARMS principle to enhance differentiation are highlighted in red.

Figure 3: Serial 10-fold dilutions of in vitro RNA standard have been tested in the corresponding real-time RT-PCR assay. Tested dilutions ranged from $1 \times 10^{8}$ to 0.1 copies/reaction for the RNA standard of lineages 1, 2, Koutango and new lineage-specific assays and consensus assay.

Figure 4: Serial 10-fold dilutions of virus in L-15 medium and human serum have been tested for consensus (panel A), lineage 1 (panel B), lineage 2 (panel C), Koutango (panel D) and new lineage (panel E) in the corresponding real-time RT-PCR assay. 


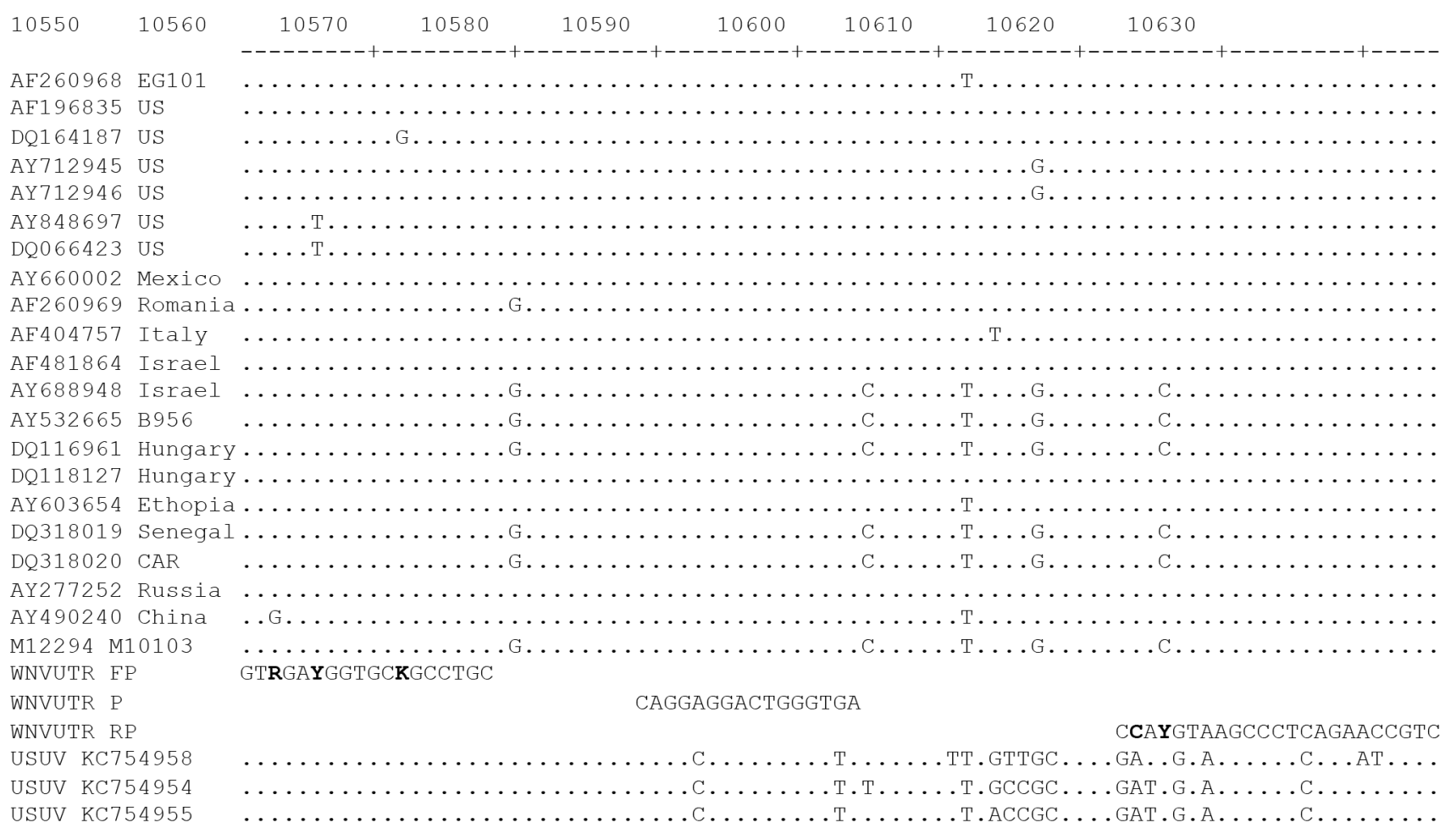




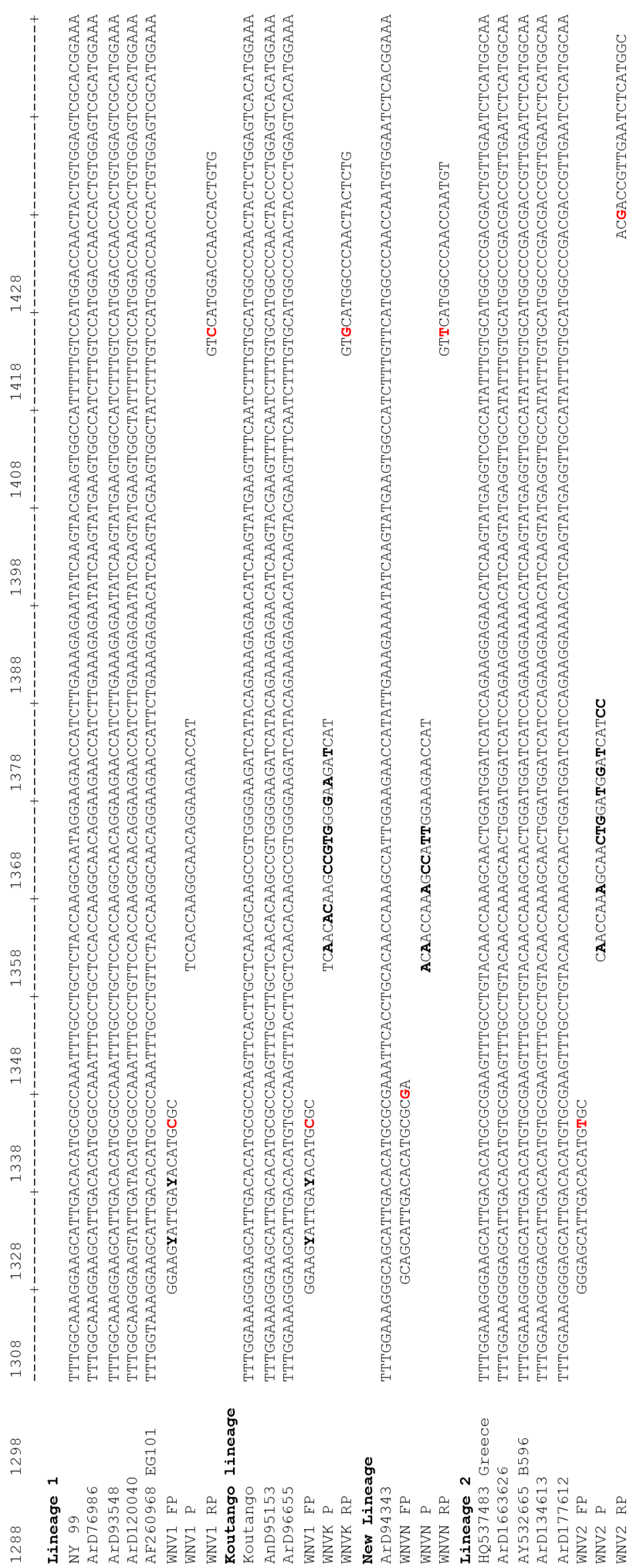




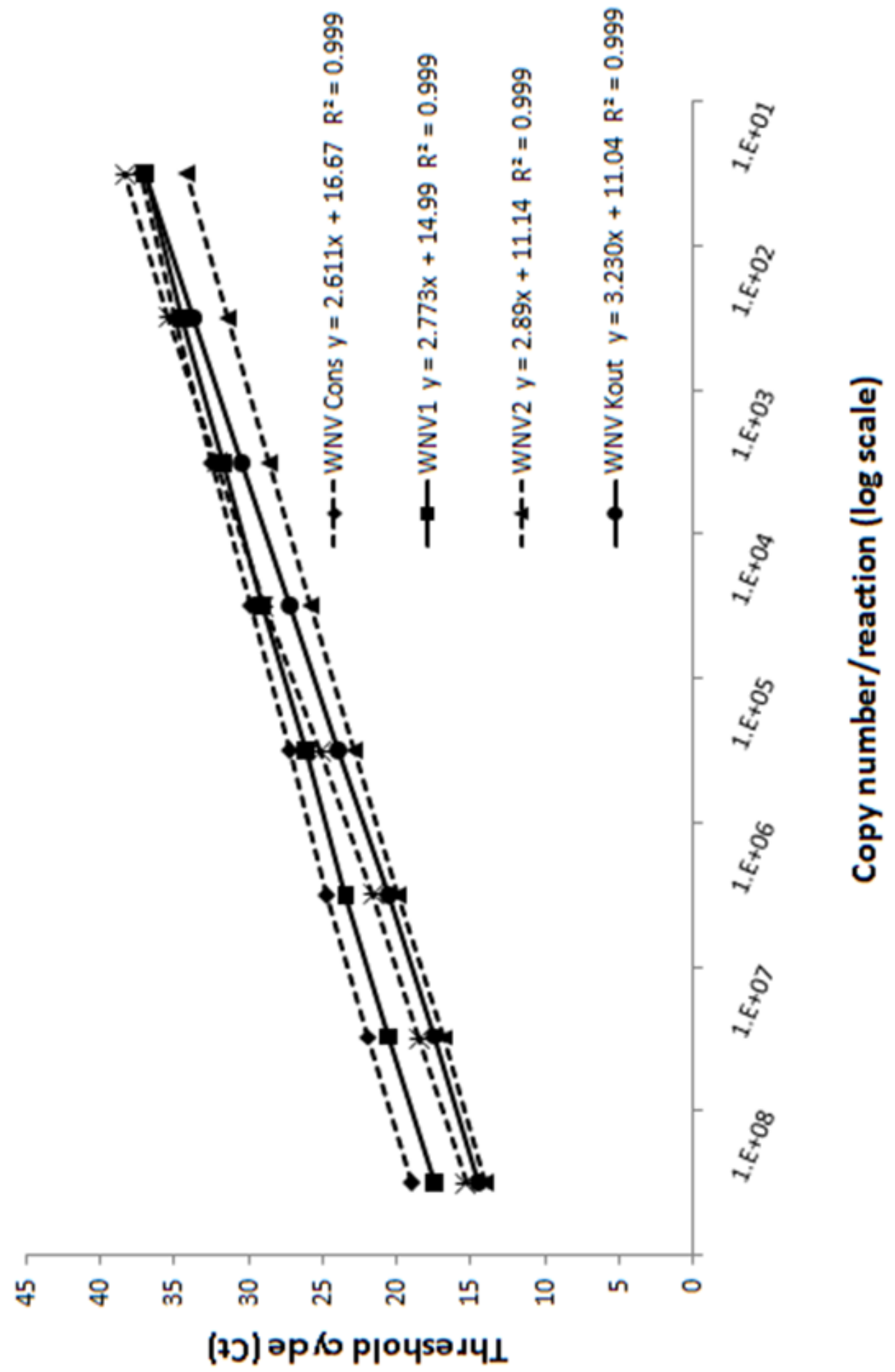




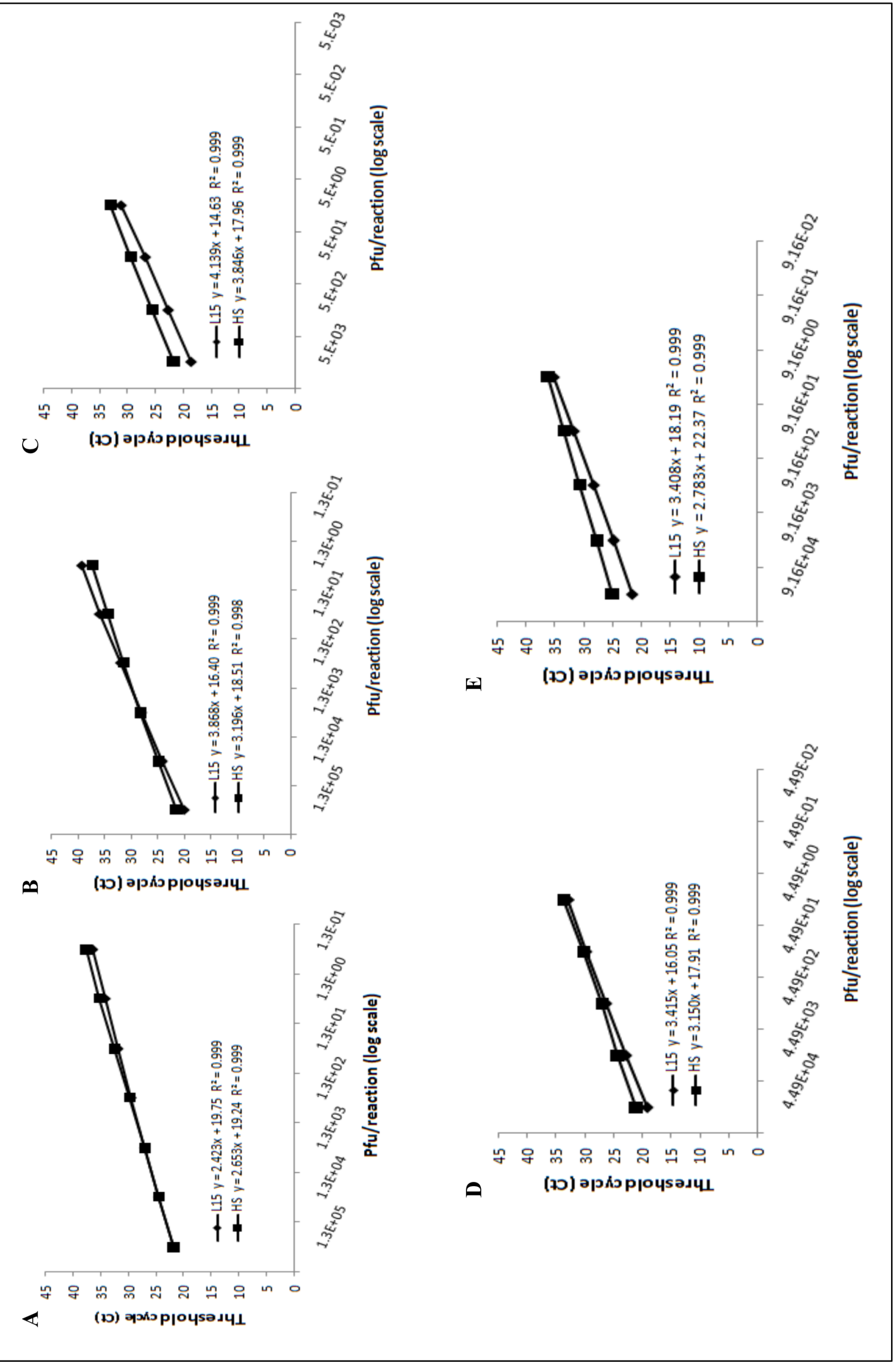

